CLINICAL STUDY

\title{
Sequential ACTH and catecholamine secretion in a phaeochromocytoma
}

\author{
P Sytze van Dam, Ad van Gils ${ }^{1}$, Marijke R Canninga-van Dijk ${ }^{2}$, Eelco J P de Koning, Leo J Hofland ${ }^{3}$ and \\ Wouter $\mathrm{W}$ de Herder ${ }^{3}$ \\ Departments of Clinical Endocrinology, ${ }^{1}$ Radiology and ${ }^{2}$ Pathology, University Medical Center, Utrecht, The Netherlands and ${ }^{3}$ Department of Internal \\ Medicine, University Hospital Dijkzigt, Rotterdam, The Netherlands \\ (Correspondence should be addressed to P S van Dam, Department of Clinical Endocrinology, University Medical Center, L00.407, PO Box 85500, \\ 3508 GA Utrecht, The Netherlands; Email: P.S.vanDam@digd.azu.nl)
}

\begin{abstract}
Objective: We describe a patient with an ACTH-producing phaeochromocytoma who initially presented with hypercortisolism and normal catecholamine concentrations, followed by near-normalisation of ACTH secretion and massive catecholamine secretion. In vitro studies were carried out on the tumour to evaluate the interaction between the tumour cells and normal adrenal cortex.

Methods and Results: A 30-year-old man initially presented with severe hypercortisolism, biochemical evidence of ectopic ACTH production, a tumour in the right adrenal gland without a hyperintense signal on the T2-weighted images at magnetic resonance imaging (MRI) scanning, and normal urinary metanephrine concentrations. After 6 months, ACTH production had almost completely resolved, but the patient developed severe hypertension and excess catecholamines. At repeated MRI-scanning, the T2-weighted images showed a hyperintense signal, in agreement with the diagnosis of phaeochromocytoma. Although the initial T1-weighted images suggested bleeding in the adrenal tumour, no signs of bleeding were observed after surgical removal. The diagnosis of ACTH-producing phaeochromocytoma was histologically and immunohistochemically confirmed. Cultured cell suspensions of the tumour secreted ACTH, which stimulated cortisol production in the ipsilateral adrenocortical cells. Conclusion: This case demonstrates that the biological activity of an ACTH-producing phaeochromocytoma can vary significantly in time, which may be the consequence of different stages of tumour differentiation.
\end{abstract}

European Journal of Endocrinology 147 201-206

\section{Introduction}

Ectopic ACTH production is a rare, but well-known, cause of Cushing's syndrome. One of the possible, but unusual, sources of ectopic ACTH production is a phaeochromocytoma, of which only a limited number of cases have been reported (1-6). We describe an unusual sequence of events in a patient with a phaeochromocytoma who first presented with severe hypercortisolism as a consequence of ectopic ACTH production, which spontaneously disappeared, whereas excess catecholamine production developed. These changes in hormone secretion were accompanied by alterations in imaging characteristics at both T1- and T2-weighted magnetic resonance imaging (MRI) scanning. After surgical removal of the phaeochromocytoma, we evaluated the in vitro ACTH secretion of cultured phaeochromocytoma cells and the direct interaction between ectopically produced ACTH and cultured cells of the ipsilateral adrenal cortex.

\section{Case report}

A 30-year-old man of Asian origin was admitted to hospital for analysis of hypercortisolism. Over a period of 1 year, he had symptoms of muscular weakness, acne, spontaneous haematomas and weight loss $(11 \mathrm{~kg})$. Furthermore, he reported heat intolerance and excessive sweating. Shortly before his admission to hospital, treatment was started for hypertension and hyperglycaemia. At physical examination, his blood pressure was $170 / 120 \mathrm{mmHg}$ and we observed a moon face, buffalo hump, central obesity, diffuse acne, a vulnerable skin and remarkable proximal muscular atrophy. Laboratory results at presentation demonstrated severe hypercortisolism, hypokalaemia, and hyperglycaemia (Table 1), for which insulin treatment was started. Plasma ACTH concentrations were greatly increased. After administration of human corticotrophin-releasing hormone (hCRH, $100 \mu \mathrm{g}$ ), plasma cortisol increased to a limited extent (baseline 1.71 
Table 1 Biochemical data at presentation and after 6 months (metyrapone was stopped 2 weeks before re-evaluation).

\begin{tabular}{lccc}
\hline & At presentation & After 6 months & Reference range \\
\hline Sodium $(\mathrm{mmol} / \mathrm{l})$ & 132 & 140 & $136-146$ \\
Potassium $(\mathrm{mmol} / \mathrm{l})$ & 3.7 & 4.6 & $3.8-5.0$ \\
Glucose $(\mathrm{not}$ fasting, mmol/l) & 24.5 & 8.9 & $3.6-8.5$ \\
$\mathrm{HbA} \mathrm{Ac}_{\mathrm{c}} \%$ & 13.6 & 0.12 & $<6.0$ \\
Midnight cortisol $(\mu \mathrm{mol} / \mathrm{l})$ & 1.33 & 50.10 \\
ACTH $(\mathrm{ng} / \mathrm{ml})$ & 425 & 277 & $20-70$ \\
Urinary free cortisol $(\mu \mathrm{mol} / 24 \mathrm{~h})$ & 21000 & 0.23 & $<0.08$ \\
Morning cortisol after $1 \mathrm{mg} \mathrm{dexamethasone}(\mu \mathrm{mol} / \mathrm{l})$ & 1.59 & 21 & $<6.0$ \\
Urinary metanephrines $(\mu \mathrm{mol} / 24 \mathrm{~h})$ & 4.4 & 150 & $8-36$ \\
Urinary VMA $(\mu \mathrm{mol} / 24 \mathrm{~h})$ & 25 & 234 & $0-100$ \\
Urinary adrenaline $(\mathrm{nmol} / 24 \mathrm{~h})$ & 244 & 93137 & $90-470$ \\
Urinary noradrenaline $(\mathrm{nmol} / 24 \mathrm{~h})$ & 2007 & 46194 & $420-2610$ \\
Urinary dopamine $(\mathrm{nmol} / 24 \mathrm{~h})$ & 17311 & & \\
\hline
\end{tabular}

and maximum $2.00 \mu \mathrm{mol} / \mathrm{l}$ ). A $7 \mathrm{~h}$ dexamethasone (7 mg iv.) infusion test showed no significant suppression of plasma cortisol concentrations (baseline 1.68 and after $7 \mathrm{~h} 1.35 \mu \mathrm{mol} / \mathrm{l})$. Therefore, Cushing's syndrome as a consequence of ectopic ACTH production was suspected. Computed tomography scanning of the chest did not reveal a potential source of ACTH. T1weighted MRI scanning of the abdomen showed bilaterally enlarged adrenal glands and a hypointense mass of $3 \times 3.5 \mathrm{~cm}$ with partial hyperintensity on the T1weighted gradient-echo image (suggestive of haematoma) in the right adrenal gland (Fig. 1A). The T2weighted images showed no hyperintense signal. An MRI scan of the pituitary gland showed no abnormalities. An indium-111-pentreotide scan (Octreoscan, Mallinckrodt, Petten, The Netherlands) and iodine- ${ }^{123} \mathrm{I}-$ metaiodobenzylguanide (MIBG) scan were negative.
The hypertension was controlled with atenolol (100 mg/day), enalapril (60 mg/day) and nifedipine $(60 \mathrm{mg} /$ day $)$. As urinary metanephrine and vanillylmandelic acid (VMA) concentrations were normal, using a routine spectrophotometric technique (7), and as the T2-weighted MRI scan and the ${ }^{123}$ I-MIBG scan were not in line with the possibility of a phaeochromocytoma, a fine-needle biopsy of the mass in the right adrenal gland was performed. This showed groups of atypical cells with anisomorphic hyperchromatic nuclei, without ACTH staining. Because the patient's clinical condition deteriorated rapidly as a consequence of persistent severe hypercortisolism, and as there was a low suspicion of the adrenal adenoma being the source of excess ACTH secretion and no immediate indication for further evaluation of the adrenal tumour, we decided to start metyrapone $500 \mathrm{mg}$ four times daily,
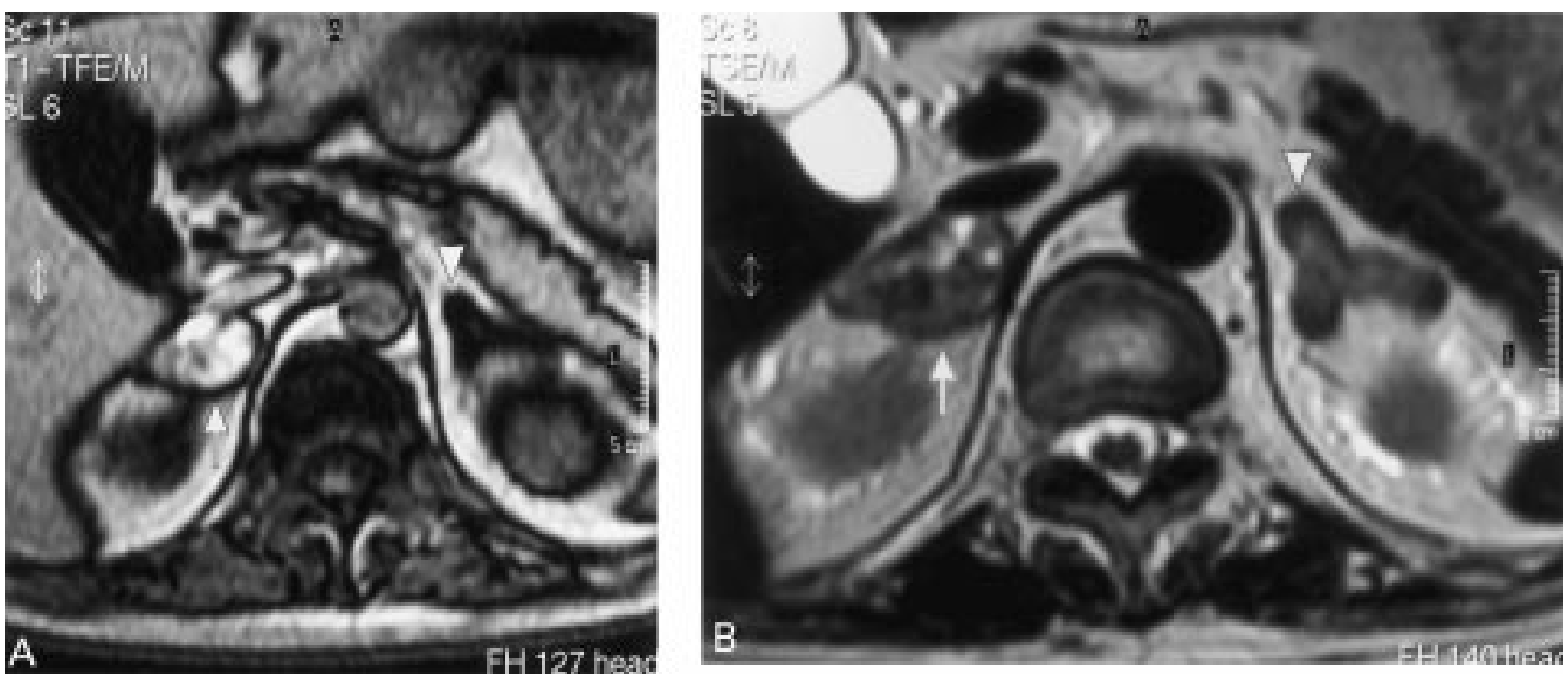

Figure $1 \mathrm{MRI}$ scan of the adrenal glands at presentation. (A) T1-weighted image (gradient-echo) showing bilateral hyperplasia of the adrenal glands (arrowhead), and a partially hyperintense mass (arrow) in the right adrenal gland. (B) T2-weighted image of the mass in the right adrenal, showing no significant hyperintense signal (arrow). 
which was followed by the gradual reduction of Cushingoid symptoms and an improvement of the patient's general condition. As no sources of ectopic ACTH production were discovered, we reconsidered the possibility of pituitary-dependent hypercortisolism, and performed bilateral sinus petrosus ACTH sampling before and after $\mathrm{CRH}$ stimulation, 2 months after presentation. Unexpectedly, basal peripheral plasma ACTH concentrations had significantly decreased to almost normal values $(47 \mathrm{ng} / \mathrm{ml})$. $\mathrm{CRH}$ stimulation resulted in a maximal central/peripheral ACTH gradient of 7.5, without lateralisation. Reassessment of urinary metanephrines and VMA showed clearly increased concentrations, and increased urinary catecholamines concentrations were found in both the initial and the new urinary samples (Table 1). The diagnosis of an
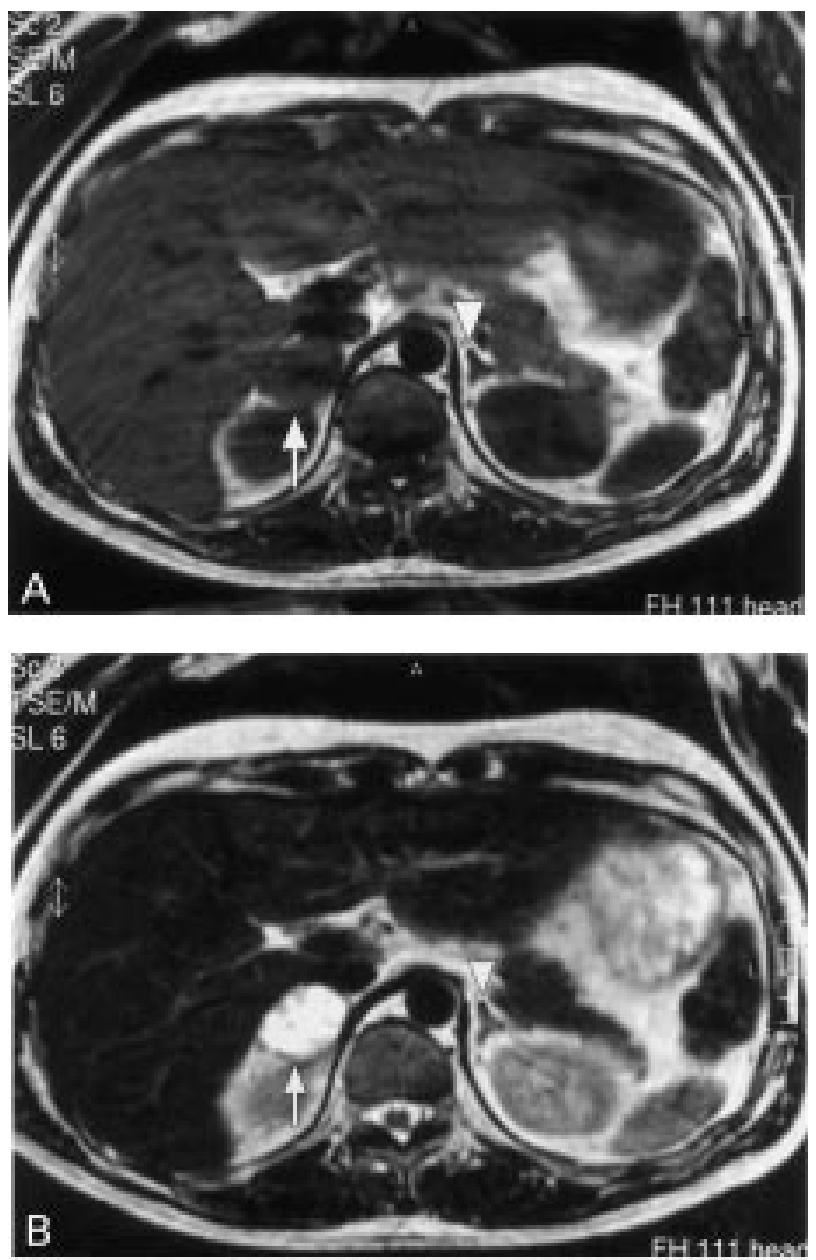

Figure $2 \mathrm{MRI}$ scan of the adrenal glands 6 months after presentation. (A) T1-weighted image (spin-echo 500, 15) showing disappearance of the bilateral adrenal hyperplasia (arrowhead), and an increase in size and reduced signal intensity of the mass (arrow) in the right adrenal gland. The dark area within the tumour reflects iron deposition. (B) T2-weighted image of the mass in the right adrenal, showing hyperintense signal (arrow).
ACTH-producing phaeochromocytoma was made, and antihypertensive treatment with the $\alpha$-blocking agent doxazosine was started. As plasma ACTH concentrations declined further, metyrapone treatment was stopped 6 months after the initial admission to hospital, and the patient remained almost normocortisolaemic (Table 1). A second MRI scan of the adrenals was performed; the T1-weighted spin-echo image showed involution of the bilateral adrenal hyperplasia, and increased size and disappearance of signal intensity of the mass in the right adrenal gland, although some signs of iron deposition could still be seen (Fig. 2A). The adrenal mass had increased to a size of $4.5 \times$ $3.5 \mathrm{~cm}$, and showed a hyperintense signal on the T2weighted image (Fig. 2B). A laparoscopic right adrenalectomy was performed. On cross section, the tumour was soft and grey-white; there were no areas suspect for haemorrhage macroscopically. Histology showed a phaeochromocytoma with positive immunostaining for ACTH, confirming the diagnosis of ACTH-producing phaeochromocytoma (Fig. 3). The distribution of the ACTH staining was not generalised, but limited to the outer rim of the tumour, where we observed three foci each with 50-60 cells strongly positive for ACTH. In the centre of the tumour, most cells did not stain, or stained only weakly, for ACTH. There were no microscopic signs of haemorrhage or infarction. Most of the tumour was analysed histologically, except for the material that was used for in vitro studies.

During the postoperative follow-up, it was possible to stop the antihypertensive treatment rapidly, and the patient recovered fully. Hydrocortisone supplementation was stopped 1 year after the operation. After 2 years of follow-up, no clinical or biochemical signs of recurrence of catecholamine or ATCH excess have been observed, which, in combination with the histological findings, indicates that the tumour was probably not malignant.

\section{In vitro studies}

After removal, normal adrenal tissue was separated from the phaeochromocytoma. The tissues were minced into small pieces and single cell suspensions of both tissues were prepared by enzymatic dissociation using collagenase, as described in detail elsewhere (8). Subsequently, the cells were resuspended in Dulbecco's modified Eagle's medium (DMEM) $+1 \%$ FCS. The phaeochromocytoma cells were incubated for 2 days at a density of 150000 cells per well per ml culture medium (DMEM $+10 \%$ FCS). After 2 days, the (conditioned) medium was collected and added directly to the cultured normal adrenal cells.

Normal adrenal cells were cultured for 2 days at a density of 160000 cells/ml culture medium (DMEM $+10 \%$ FCS $)$ per well in multiwell plates. After 2 days, the medium was replaced by DMEM $+1 \%$ 


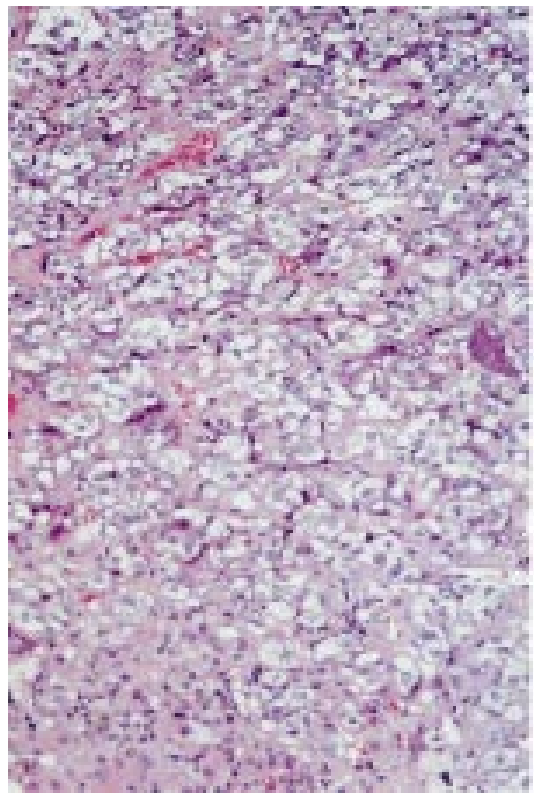

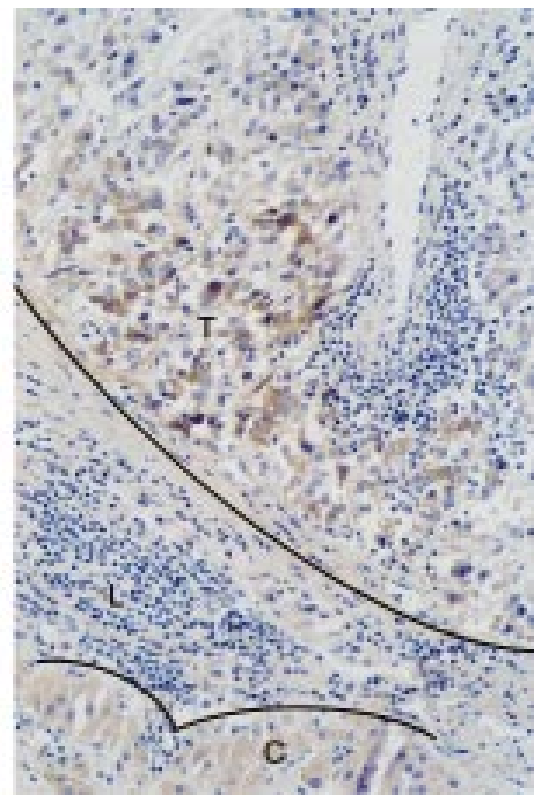

Figure 3 Histological features of the tumour. Left: Phaeochromocytoma cells with fine granular cytoplasm and presence of nuclear pleomorphism. Right: Positive staining for ACTH in the periphery of the tumour. $\mathrm{T}$, tumour; $\mathrm{C}$, adjacent adrenal cortex; L, lymphoid infiltrate.
FCS, followed by an additional 24-h incubation. After this preincubation, the medium was refreshed and a 2 -h incubation in DMEM $+10 \%$ FCS was performed in triplicate, without or with $123 \mathrm{pg} / \mathrm{ml} \mathrm{ACTH}_{1-24}$ (Synacthen, Ciba-Geigy, Basel, Switzerland) or with $1 \mathrm{ml}$ per well of the undiluted conditioned (2 days) medium from the phaeochromocytoma cells. At the end of the incubation, medium was collected for determination of cortisol concentration. The conditioned medium of the phaeochromocytoma cells contained $138 \pm 5 \mathrm{pg} / \mathrm{ml}$ ACTH (mean \pm s.D., $n=3$ ).

\section{Results}

Figure 4 shows that synthetic $\mathrm{ACTH}_{1-24}$ at a concentration of $123 \mathrm{pg} / \mathrm{ml}$ significantly stimulated cortisol production, by $149 \%(P<0.05$ compared with control cells). In addition, conditioned medium from the phaeochromocytoma cells (containing $138 \mathrm{pg} / \mathrm{ml}$ ACTH), significantly stimulated cortisol production, by $47 \%$ $(P<0.05$ compared with control cells), suggesting that the ACTH in the conditioned medium of the phaeochromocytoma cells was bioactive. The observation that the ACTH of the conditioned medium induced a lesser stimulation of cortisol production than did a comparable concentration of synthetic $\mathrm{ACTH}_{1-24}$ might be explained by the possibility that some degradation of the ACTH in the conditioned medium has occurred during the 2-day culture period.

\section{Discussion}

Cushing's syndrome as a consequence of ACTH-producing phaeochromocytoma has been described previously, but the number of reported cases is limited $(1,3-6,9)$. In a recent survey of 1096 adrenal incidentalomas, this diagnosis was not reported (10). In patients with proven ectopic ACTH production, frequencies of ACTH-producing phaeochromocytoma between 0 and $12 \%$ have been reported (11). The present case is unusual, both because of the spontaneous remission of severe hypercortisolism that occurred concomitantly with an important increase in catecholamine and dopamine production, and because of the different aspects of the adrenal at MRI scanning, which could represent differences in metabolic activity.

Spontaneous normalisation of ACTH production in a patient with an ACTH-producing phaeochromocytoma has been described once previously (5). There is a striking resemblance between our patient and that patient a 25-year-old female who presented with Cushing's

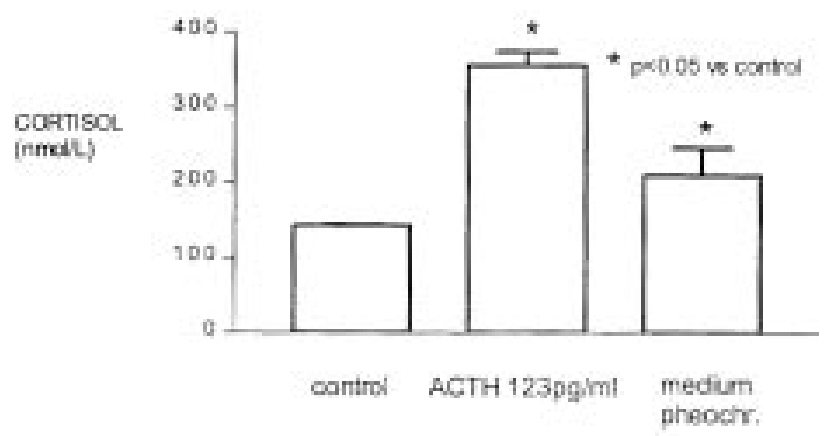

Figure 4 Cortisol secretion by adrenal cell suspensions from the ipsilateral adrenal cortex after incubation with a control solution, a solution containing biochemically synthesised ACTH, and the undiluted conditioned medium after 2 days of incubation of the phaeochromocytoma cells. 
syndrome, ectopic ACTH production, normal urinary VMA and increased urinary catecholamine concentrations. The hypercortisolism in that patient went into complete remission after initial pharmacological treatment with ketoconazol was stopped. She developed symptoms of excess catecholamine production after 18 months, which necessitated surgical removal of the tumour. Histology also showed positive immunostaining for ACTH. According to the results of 24-h urinary free cortisol concentrations and the 1-mg overnight dexamethasone suppression test, the remission of hypercortisolism in our patient was not complete, but clinically highly significant.

We cannot exclude the possibility that our patient had cyclic Cushing's syndrome. This has been described once in a patient with an ACTH-secreting phaeochromocytoma (4), who showed spontaneous normalisation of cortisol secretion over a period of 4 months, which was followed by a relapse of hypercortisolism necessitating adrenalectomy. In our patient, there was an interval of at least 5 months between the normalisation of ACTH concentrations and the time of operation, when the patient was still clinically and biochemically almost eucortisolaemic.

The cause of the disappearance of hypercortisolism in our patient and in the two previously reported patients $(4,5)$ is unclear. Imaging studies of the adrenal adenoma using T1-weighted MRI scanning suggested that haemorrhage had occurred. However, no macroor microscopic evidence of haemorrhage was observed in the tumour after resection. Furthermore, catecholamine secretion dramatically increased in the months after the initial MRI scan and the biopsy; it is highly unlikely that bleeding in the tumour would selectively affect the ACTH-producing cells. Therefore, it remains unclear why this hyperintense signal on the T1 image was observed, although spontaneous bleeding remains the most probable explanation, in particular because the gradient-echo technique, which is the most sensitive technique for the detection of hemorrhage, was used.

The absence of high signal intensity on the initial T2weighted MRI images, the negative ${ }^{123} \mathrm{I}$-MIBG scan, and the normal first urinary metanephrine and VMA excretion were three reasons for us initially to reject the possibility of a phaeochromocytoma as the source of the ACTH excess and, in combination with the clinical condition of the patient, not to perform adrenalectomy. High signal intensity on the T2-weighted image is considered pathognomonic for phaeochromocytoma. Its sensitivity for the diagnosis of phaeochromocytoma was reported to be $100 \%$ in a study with a limited number of patients (12). It is suggested that the variable secretion of catecholamines by phaeochromocytomas is correlated with the intensity of T2weighted images. However, only limited data regarding such an association have been reported (13). The reason for the negative ${ }^{123} \mathrm{I}-\mathrm{MIBG}$ scan may be that it was performed at an early stage when ACTH production was predominant and catecholamine secretion low.

The decrease in ACTH secretion might be explained by a change in tumour differentiation or endocrine activity accompanied by a decrease in pro-opiomelanocortin gene transcription $(14,15)$. The synthesis of adrenaline depends upon the activity of the enzyme phenylethanolamine- $N$-methyltransferase (PNMT). The enzymatic activity of PNMT is enhanced by increased ambient cortisol concentrations (16). Furthermore, some studies show that the activity of tyrosine hydroxylase (the enzyme converting tyrosine to dopa and thus contributing to the synthesis of the noradrenaline and adrenaline precursor, dopamine) is stimulated by ACTH (17). The T2 images suggest that increased signal reflects increased catecholamine content, which was also biochemically proven. It can be argued that this change in signal characteristics reflects a change in the degree of tumour differentiation. The changes in adrenaline/total catecholamine urinary ratio that we observed are suggestive of tumour dedifferentiation (18). However, as we could not perform tumour cell cultures in the early stages of the course of the disease, the possibility of tumour (de)differentiation and the underlying mechanism remains speculative. Therefore, alternative hypotheses, such as the presence of two different pools of tumour cells with different secretory differentiation and with intermittent ACTH secretion, cannot be excluded.

In our patient, an important increase in secretion of noradrenaline was observed 6 months after the initial presentation. The discrepancy between metanephrine and catecholamine secretion demonstrates that, although the sensitivity of urinary metanephrine concentrations for the detection of phaeochromocytoma is reported to be more than $95 \%(19,20)$, it is not a fully reliable test. It should be repeated in case of doubt, and additional assessment of urinary catecholamines or plasma concentrations of metanephrines, catecholamines, or both, should be performed in case of serious clinical suspicion of a phaeochromocytoma. As this discrepancy was observed both in our patient and in the patient reported by Loh et al. (5), it can be speculated that hypercortisolism or ectopic ACTH production negatively affects the sensitivity of urinary metanephrine excretion, although the mechanism is unclear.

Our in vitro data demonstrate that the adenoma cells actively secreted ACTH and that this ACTH was biologically active. Probably, more ACTH secretion would have been observed if the tumour had been removed at an earlier stage, judging from the marked disappearance of clinical and biochemical features of hypercortisolism in this patient. Because symmetric bilateral adrenocortical hyperplasia was observed in our patient, as in most other patients with ACTHproducing phaeochromocytoma, we believe that there 
is no direct paracrine effect of the ectopic ACTH on the adrenal glands. The correlation between high plasma concentrations of ACTH and hypercortisolaemia tends to indicate a classical endocrine mechanism.

In summary, the present case report demonstrates that the biological activity of an ectopic ACTH-producing phaeochromocytoma can vary significantly. The mechanism responsible for this phenomenon is still unexplained, but alterations in tumour differentiation probably play a part. The alterations in MRI findings possibly reflect this change in tumour differentiation. Unfortunately, the low incidence of ACTH-producing phaeochromocytoma and the variation in clinical presentation preclude extensive research on the pathogenesis and natural evolution of this condition.

\section{Acknowledgements}

We thank P M van Koetsveld for his expert technical assistance in carrying out the in vitro studies, and Professor C J M Lips for his critical comments on the first version of the manuscript.

\section{References}

1 Sato M, Watanabe T, Ashikaga T, Taneda T, Yamawake N, Nishizaki $\mathrm{M}$ et al. Adrenocorticotropic hormone-secreting pheochromocytoma. Internal Medicine 199837 403-406.

2 Forman BH, Marban E, Kayne RD, Passarelli NM, Bobrow SN, Livolsi VA et al. Ectopic ACTH syndrome due to pheochromocytoma: case report and review of the literature. Yale Journal of Biological Medicine 197952 181-189.

3 Chen H, Doppman JL, Chrousos GP, Norton JA, Nieman LK \& Udelsman R. Adrenocorticotropic hormone-secreting pheochromocytomas: the exception to the rule. Surgery $1995 \mathbf{1 1 8}$ 988-994.

4 Terzolo M, Ali A, Pia A, Bollito E, Reimondo G, Paccotti P et al. Cyclic Cushing's syndrome due to ectopic ACTH secretion by an adrenal pheochromocytoma. Journal of Endocrinological Investigation 199417 869-874.

5 Loh KC, Gupta R \& Shlossberg AH. Spontaneous remission of ectopic Cushing's syndrome due to pheochromocytoma: a case report. European Journal of Endocrinology 1996135 440-443.

6 White A, Ray DW, Talbot A, Abraham P, Thody AJ \& Bevan JS. Cushing's syndrome due to phaeochromocytoma secreting the precursors of adrenocorticotropin. Journal of Clinical Endocrinology and Metabolism 200085 4771-4775.

7 Stroes JW, Putters J \& van Rijn HJ. An improved spectrophotometric procedure for the determination of urinary metanephrines.
Journal of Clinical Chemistry and Clinical Biochemistry 198725 483-486.

8 Marzouk HF, Hofland LJ, den Holder FH, van Koetsveld PM, Steenbergen J, Zuiderwijk J et al. Effects of suramin on hormone release by cultured rat anterior pituitary cells. Molecular and Cellular Endocrinology 199072 95-102.

9 Liu J, Heikkila P, Voutilainen R, Karonen SL \& Kahri AI. Pheochromocytoma expressing adrenocorticotropin and corticotropin-releasing hormone: regulation by glucocorticoids and nerve growth factor. European Journal of Endocrinology 1994 $131221-228$.

10 Mantero F, Terzolo M, Arnaldi G, Osella G, Masini AM, Ali A et al. A survey on adrenal incidentaloma in Italy. Study Group on Adrenal Tumors of the Italian Society of Endocrinology. Journal of Clinical Endocrinology and Metabolism 200085 637-644.

11 Becker M \& Aron DC. Ectopic ACTH syndrome and CRH-mediated Cushing's syndrome. Endocrinology and Metabolism Clinics of North America 199423 585-606.

12 Peplinski GR \& Norton JA. The predictive value of diagnostic tests for pheochromocytoma. Surgery 1994116 1101-1109.

13 Takeda M, Katayama Y, Takahashi H, Terunuma M, Nishiyama T, Sato S et al. MRI of pheochromocytoma - comparison with CT, 131I-MIBG, urinary catecholamine level and operative findings. Nippon Hinyokika Gakkai Zasshi 199283 33-39.

14 Baylin SB \& Mendelsohn G. Time-dependent changes in human tumors: implications for diagnosis and clinical behavior. Seminars in Oncology 19829 504-512.

15 White A \& Clark AJ. The cellular and molecular basis of the ectopic ACTH syndrome. Clinical Endocrinology 199339 131141.

16 Betito K, Diorio J, Meaney MJ \& Boksa P. Adrenal phenylethanolamine N-methyltransferase induction in relation to glucocorticoid receptor dynamics: evidence that acute exposure to high cortisol levels is sufficient to induce the enzyme. Journal of Neurochemistry 199258 1853-1862.

17 Valenta LJ, Elias AN \& Eisenberg H. ACTH stimulation of adrenal epinephrine and norepinephrine release. Hormone Research 1986 23 16-20.

18 Plouin PF, Chatellier G, Fofol I \& Corvol P. Tumor recurrence and hypertension persistence after successful pheochromocytoma operation. Hypertension 199729 1133-1139.

19 Eisenhofer G, Lenders JW, Linehan WM, Walther MM, Goldstein DS \& Keiser HR. Plasma normetanephrine and metanephrine for detecting pheochromocytoma in von Hippel-Lindau disease and multiple endocrine neoplasia type 2. New England Journal of Medicine 1999340 1872-1879.

20 Heron E, Chatellier G, Billaud E, Foos E \& Plouin PF. The urinary metanephrine-to-creatinine ratio for the diagnosis of pheochromocytoma. Annals of Internal Medicine 1996125 300-303.

Received 3 December 2001

Accepted 3 April 2002 